\title{
FAST DIGITAL ORBIT FEEDBACK SYSTEMS AT NSLS*
}

\author{
B. Podobedov ${ }^{\dagger}$, B. Kushner, S. Ramamoorthy, Y. Tang, and E. Zitvogel \\ National Synchrotron Light Source, Brookhaven National Laboratory, Upton, NY 11973
}

\begin{abstract}
We are implementing digital orbit feedback systems to replace the analog ones in both the VUV and the X-ray rings. We developed an original VME-based design which is run by a powerful Motorola 2305 CPU and consists entirely of off-the-shelf VME boards. This makes the system inexpensive and easy to configure, and allows for high digitizing rates. The new $5 \mathrm{kHz}$ digital global feedback system is currently operational in the VUV ring, and the X-ray system is in the commissioning phase. Some of the parameters achieved include vertical correction bandwidth of $200 \mathrm{~Hz}$ (at DC gain of 100) and typical orbit drift over a fill of $<3 \%$ of the rms beam size. In this paper we discuss the system architecture, implementation and performance.
\end{abstract}

\section{HISTORY AND MOTIVATION}

NSLS is a synchrotron light user facility providing radiation to a large number of the X-ray and VUV ring users. Although both rings generally provide high quality beam, as with any accelerator, they are prone to environmental noise creating a nuisance to some users. Main sources of noise include NSLS and AGS booster operation, floor vibrations due to water pumps and other equipment, and powerline noise at harmonics of $60 \mathrm{~Hz}$. There is also a gradual orbit drift over a fill mainly caused by the mechanical motion of ring components under variable heat load. To combat these, from very early on, the NSLS had a vigorous program to improve orbit stability. In the early $80 \mathrm{~s}$, local feedbacks based on analog electronics were put in all of the X-ray ring insertion device beamlines [1]. Later, a global orbit correction principle was pioneered at NSLS [2] which eventually resulted in analog global feedback system installation in both the X-ray and VUV rings [3].

While generally providing good stability, present analog systems have some limitations. Most importantly, they use a limited number of BPMs and corrector magnets (trims). This results in good control only at certain locations of each ring. Since the analog design is not easily scalable, it would be an enormous effort to expand the system. In addition, analog architecture is not flexible enough in the choice of system configuration, correction algorithms, filters, etc.

With the progress in digital electronics it was realized that a digital design can effectively address all of these issues. A prototype global digital feedback system operating at $550 \mathrm{~Hz}$ was built and tested at the X-ray ring, proving a clear advantage of a digital design [4]. It demonstrated a significant reduction in the long term orbit drift compared

\footnotetext{
* Work supported by US DOE under contract DE-AC02-98CH10886

†E-mail: boris@bnl.gov
}

to the analog systems in operation, as well as a much better correction of $1.2 \mathrm{~Hz}$ harmonics of NSLS booster noise. However, due to its low sampling rate this "old digital system" could not match the bandwidth of the analog systems and it was never put to regular operation.

This is why, as the next step in improving orbit stability, we decided to build a much faster digital orbit feedback system. To date both the X-ray and the VUV ring systems are built and the VUV system is in regular operations. In the rest of this paper we describe the system architecture, implementation, performance and operating experience.

\section{DESIGN AND IMPLEMENTATION}

\subsection{System Design Trade-Offs}

The analog systems had a bandwidth of $\sim 120 \mathrm{~Hz}$ [3], limited by the $30-60 \mathrm{~Hz}$ bandwidth of the correctors installed outside of the $4 \mathrm{~mm}$ wall thick aluminum vacuum chamber. To match this bandwidth without extreme complexities in the digital controller the sampling rate should be 30-50 times higher [7]. The chosen rate of $5 \mathrm{kHz}$ meets this criteria. Also, running at this frequency does not require extra anti-aliasing filters since the existing NSLS $\mathrm{BPM}$ receivers have $2 \mathrm{kHz}$ low-pass filters at the output.

Some of the functions needed for a feedback system (orbit sampling, trim control, etc.) have been implemented for a long time in the so-called orbit and trim micros, used mainly in manual orbit corrections. It was tempting to build a very compact feedback system communicating with these two micros with its own sole function of calculating required corrector strengths. This is, in fact, how it was done (using shared memory) in the old digital system at NSLS. However, we found that for existing hardware and a typical data volume needed, communication between the micros could not sustain a $5 \mathrm{kHz}$ rate. Rather than upgrading existing micros, we decided to build an independent system with its own digitizers and communication with the trims. Apart from high system rate, this redundancy allowed us a much faster development since we didn't have to modify the hardware used in regular operations.

We considered an option of digitizing right in the BPM receivers using custom-made proprietary boards from an outside manufacturer. Although this approach could provide high noise immunity, we were concerned with potential radiation damage to the digitizers, since, in the X-ray ring, they would have been inside the tunnel. We ended up using existing analog lines from the receivers to the orbit micro (which we have buffered on the micro end) and then using off-the-shelf VME ADC boards for digitization.

Finally, there was the choice of system architecture. Other light sources mostly use DSPs which perform both 
the I/O functions, as well as the corrector strength calculations [5]-[6]. Due to our expertise with VME systems we opted for a single board VME processor running the $\mathrm{Vx}$ Works real time operating system to do the feedback calculations. The I/O part is performed in commercially available VME boards, with all the internal DSP programming done by the manufacturer.

\subsection{System Layout and Correction Principles}

Based on these considerations we have designed and built three separate systems, one for the VUV ring, correcting beam motion in both horizontal and vertical planes, and one per plane in the X-ray ring. Each system occupies a single VME crate and all three are very similar except for a number of the I/O boards used. The conceptual system layout is shown in Fig. 1.

Buffered BPM receiver signals are digitized by ADC boards and, based on these readings, a CPU calculates the corrector strengths. These are subsequently converted to an analog form in the DAC boards connected to trim power supplies. To perform a basic feedback cycle this system doesn't need to communicate with the slow parts of the NSLS control system and may run at a very high rate. Using orbit signal buffers and summing junctions at the trim power supplies left the rest of the control system intact, allowing us seamless switch-overs between the study runs with the digital feedback turned on and regular operations.

To calculate the trim settings at each feedback cycle we use an SVD response matrix inversion algorithm which is now widely used in real time orbit feedback systems [3][6]. To make the closed loop system stable, the SVD correction is calculated after applying a digital filter to the measured orbit which sets the system gain and defines the dynamic behavior. In the VUV ring we are presently using a simple IIR filter that performs basic lag compensation for slower trim response at higher frequencies. We are also experimenting with higher order filters in the X-ray ring.

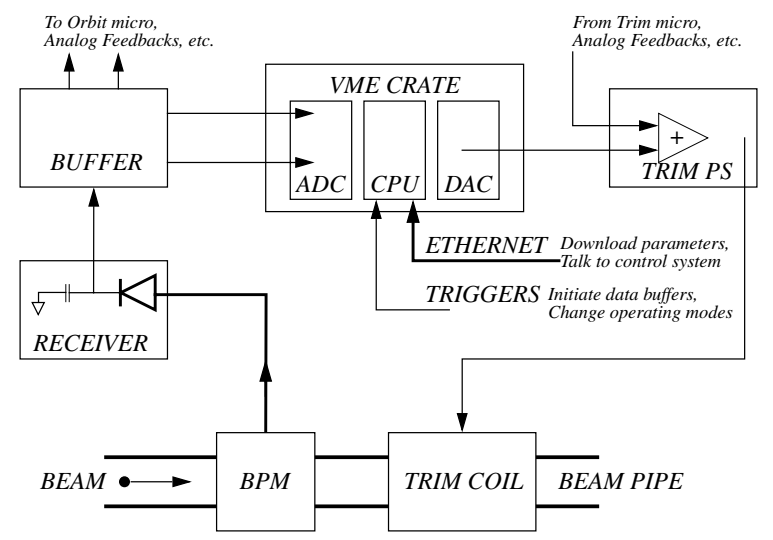

Figure 1: System layout.

\subsection{Hardware Details and Configuration}

The system uses 16 bit VMIVME-3123 ADC and VMIVME-4116 DAC boards manufactured by VMIC. The ADCs support up to $100 \mathrm{kHz}$ simultaneous data acquisition on all 16 channels. The 8 channel DACs have $10 \mu \mathrm{s}$ settling time. For compatibility with other NSLS micros, we decided to keep a Power-PC CPU and use an MVME 2305 model with $300 \mathrm{MHz}$ system clock. Upgrades are possible should we require more computing power.

The number of I/O boards in each system is defined by the total number of BPMs and correctors in each ring. The VUV ring has 24 BPMs and 16 trims per plane, so the feedback system has $3 \mathrm{ADC}$ and $4 \mathrm{DAC}$ boards. In the X-ray ring there are 39 vertical and 55 horizontal correctors and 48 BPMs in each plane, so we planned on using 5 DACs in the vertical and 7 DACs in the horizontal systems plus 3 ADCs in each. Recently we added an extra ADC per plane that samples additional signals including a high resolution photon beam position detector. The latter is used in one of the analog local feedbacks which will be soon incorporated in the digital system.

While for both rings a working digital feedback configuration typically includes all the BPMs, it may not include all the trims. For example, the VUV ring system operates with 24 BPMs and 8 trims for each plane using 8 eigenvectors for correction. In this configuration $48 \mu$ s of CPU time is spent getting orbit data from the ADC memory and writing kick values to the DACs, and $88 \mu$ s are spent calculating the corrections, leaving extra $64 \mu$ s for each cycle. In the X-ray ring, which has two independent systems, we can use up to 16 eigenvectors (with all BPMs and correctors enabled) before we run into CPU constraints.

\section{SYSTEM PERFORMANCE}

\subsection{High Frequency Noise Reduction}

To illustrate the system performance in reducing the orbit noise we present a typical spectrum of a vertical X-ray BPM with and without the feedback (Fig. 2). At the DC

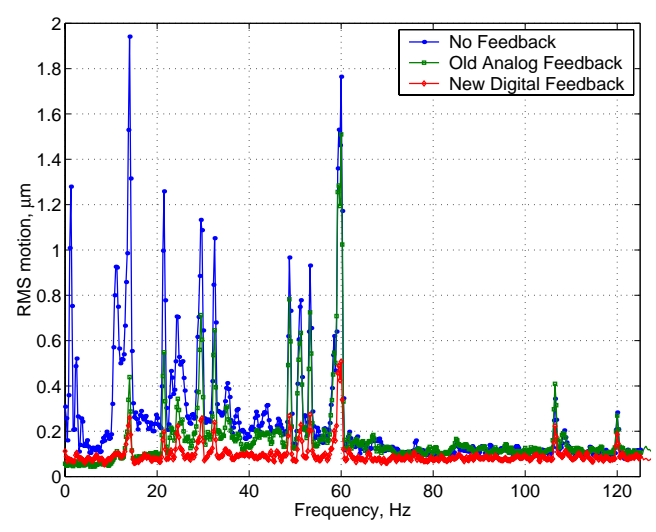

Figure 2: Typical noise reduction on a vertical BPM.

gain of 100 the system virtually eliminates the low fre- 
quency noise while the $60 \mathrm{~Hz}$ and $120 \mathrm{~Hz}$ power line noise components are reduced by factors of 3.5 and 1.5. A driven beam response measurement performed for this configuration gives the $0 \mathrm{~dB}$ correction bandwidth of about $200 \mathrm{~Hz}$.

For comparison we also plot the same signal with the analog feedback on. While the two systems provide similar noise reduction at low frequencies, as the frequency goes up the analog system runs out of gain much faster, providing, for example less than $20 \%$ improvement in the $60 \mathrm{~Hz}$ line. Note, that the signal plotted in Fig. 2 was measured at one out of 16 BPMs included in the global analog system. A similar comparison performed at one of the BPMs that is not in the analog feedback, would show an even bigger advantage for the digital, since the analog system may increase the orbit noise at those BPMs.

\subsection{Long Term Drift Reduction}

Another benefit of the new system is a greatly reduced slow orbit drift. The amount of drift may differ a lot around the ring, so, to illustrate this benefit, we plot two histograms of the drift values at all 24 vertical BPMs in the VUV ring (Fig. 3). The drift is defined as the difference of two extreme BPM readings over a 5 hour operational fill. The median drift value with no feedback is $35 \mu \mathrm{m}$ and with the feedback on it is reduced by a factor of 5 (vs. 2 for the ana$\log$ system) bringing the total drift to $<3 \%$ of the FWHM beam size. A similar percentage was also achieved for the horizontal plane, so further drift reduction is hardly needed.

Similarly to the VUV ring, the new X-ray ring feedbacks significantly reduce the orbit drift. However, there is more work to be done, since a substantial part of the measured drift in the horizontal plane is due to the motion of the vacuum chamber (that holds pickup electrodes) under varying thermal load [8]. Unless the true orbit motion is measured, correcting the drift may be of no benefit to the users.

We are now measuring chamber motions at various locations and correlating them with beam current and local chamber temperatures. In the future this will let us adjust the orbit measurement using a lookup table or a real time chamber position measurement before computing the feedback correction. As a short term solution we plan to add another filter to the digital algorithm, so that the corrections corresponding to higher eigenvectors (more sensitive to isolated BPM errors) are tapered off at very low frequencies.
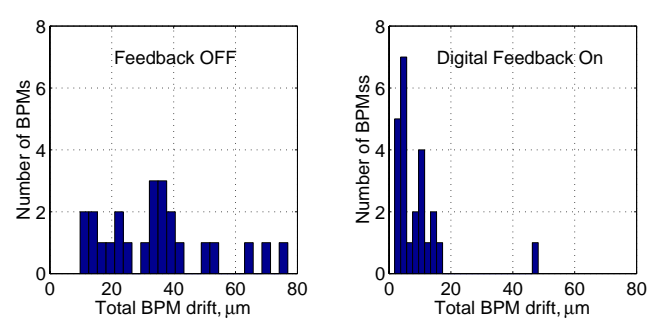

Figure 3: VUV vertical orbit motion over a 5 hour fill.

\subsection{Machine Diagnostics}

Similar to other facilities, we found digital feedback to be a valuable diagnostics tool. A ring buffer set in the on-board CPU memory stores orbit readings for all BPMs and, when the feedback is on, also stores the trim corrections. Presently we use a large enough buffer to hold 1 second worth of data sampled at $5 \mathrm{kHz}$. The ring buffer may be stopped at any time by either a software or a hardware interrupt, with the latter connected to the ring's "beam dump" trigger. Once stopped, the buffer may be downloaded for analysis. Since the sampling frequency is orders of magnitude higher than is provided in orbit and trim micros, plotting the buffer data in time or frequency domain quickly helps us to troubleshoot many problems with the ring that used to be solved by extensive analog measurements. These include identifying malfunctioning trims or BPMs and pinpointing the sources of orbit disturbance.

\section{SUMMARY AND OUTLOOK}

We have designed and built a $5 \mathrm{kHz}$ digital orbit feedback system. The system is inexpensive and was built with minimal modifications to the rest of the NSLS machine. The system provides substantial orbit noise reduction up to $200 \mathrm{~Hz}$, reduces long term orbit drift and is effectively used for machine diagnostics.

Our next goal is to put the X-ray ring system in regular operations. This includes modifying the correction algorithms (like introducing BPM weights) so that it replaces not only the analog global system but also the numerous local feedbacks. Additional issues include systematic BPM errors due to slow horizontal motion. We are also working on modeling the system using Matlab ${ }^{\odot}$ family software, to design more effective digital algorithms which will provide further improvement to the orbit correction.

\section{ACKNOWLEDGEMENTS}

We wish to thank Rich Biscardi, Sam Krinsky, Rich Michta and John Smith of NSLS, and Om Singh of APS, ANL, for stimulating discussions. This work is supported by the US DOE under contract DE-AC02-98CH10886.

\section{REFERENCES}

[1] R.J. Nawrocky et al, Proc 1989 PAC, 1856.

[2] L.H. Yu et al, NIM A 284 (1989), 268.

[3] O. Singh, Y. Tang, S. Krinsky, NIM A 418 (1998), 267.

[4] A. Friedman, E. Bozoki, NIM A 352 (1994), 339.

[5] J. Carwardine, F. Lenkszus, AIP Conf Proc 451 (1998), 125.

[6] E. Plouviez, J.M. Koch, F. Uberto, Proc ICALEPCS'99, 275.

[7] G. Franklin, J. Powell, M. Workman, "Digital Control of Dynamic Systems" Addison Wesley Longman, 3rd edtn. (1997).

[8] L. Solomon, D. Lynch, J. Safranek, Proc 1999 PAC, 2244. 